\title{
Gene expression profiling in peripheral blood leukocytes as a new approach for assessment of human stress response
}

\author{
Kazuhito Rokutan ${ }^{1,3,4}$, Kyoko Morita ${ }^{1,3}$, Kiyoshi Masuda', Kumiko Tominaga', \\ Michiyo Shikishima ${ }^{4}$, Shigetada Teshima-Kondo ${ }^{1,3}$, Tetsuro Omori ${ }^{2,3}$, and Atsuo Sekiyama ${ }^{1}$ \\ ${ }^{1}$ Department of Stress Science, and ${ }^{2}$ Department of Psychiatry, Institute of Health Biosciences, The University of \\ Tokushima Graduate School, Tokushima; ${ }^{3}$ Human Stress Research Center, University of Tokushima; \\ and ${ }^{4}$ The Research Institute of Science and Technology for Science of Japan Science and Technology, \\ Tokyo, Japan
}

\begin{abstract}
Stress is the coordinated physiological processes to maintain a dynamic equilibrium under stressful conditions. The equilibrium is threatened by certain physiological and psychological stressors. Stressors trigger physiological, behavioural, and metabolic responses that are aimed at reinstating homeostasis. The hypothalamus-pituitary-adrenal (HPA) axis and the sympathetic nervous system play an essential role in the stress response. Excessive, prolonged, or inadequate response that is termed as "allostasis" or "allostatic load" leads to pathological outcomes. Dysregulation of the HPA axis activity is involved in the pathogenesis of stressrelated disorders including major depression. The complex brain-immune-endocrine network regulates the HPA axis, and hereditary predisposition as well as environmental factors such as traumatic experiences in early life also modifies the capacity of an individual to cope. Therefore, it is difficult to correctly assess the complex stress response. We have developed a microarray carrying 1,467 cDNAs that were selected to specifically measure stress response in peripheral blood leukocytes. Using this tool, we have succeeded to objectively assess individual response to acute psychological stress and to detect unique expression profiles in patients with depression. Gene expression profile in peripheral blood leukocytes may be a potentially useful for the detection of disease-associated, abnormal stress responses. J. Med. Invest. 52 : 137-144, August, 2005
\end{abstract}

Keywords : stress, allostasis, hypothalamus-pituitary-adrenal axis, stress assessment, microarray

\section{INTRODUCTION}

\section{Concept of stress}

All living organisms strive towards a dynamic equilibrium that is called homeostasis. Cannon first introduced the term "homeostasis" to describe this coordinated physiological processes (1). For maintenance of the steady states in the organism, he mainly focused on the sympathetic nervous system as an essential homeostatic one that serves to restore stress-

Received for publication July 8, 2005 ; accepted July 15, 2005.

Address correspondence and reprint requests to Kazuhito Rokutan, M.D., Ph.D., Department of Stress Science, Institute of Health Biosciences, The University of Tokushima Graduate School, 3-18-15 Kuramoto-cho, Tokushima 770-8503, Japan and Fax :+81-88-6339008. induced disturbed homeostasis and to promote survival of the organism. Selye introduced the term "stress" as a medical and scientific idea (2), describing a pathological triad (adrenal enlargement, gastrointestinal ulceration, and thymicolymphatic involution) caused by a variety of stressors. From this pathological triad, Selye developed a theory of stress that aroused intense research interest. He defined stress as the nonspecific response (revealed after subtraction of the specific components from the total response) of the body to any demand, emphasizing that the same pathological triad "stress syndrome", which would result from exposure to any stressor. In contrast to Cannon, Selye mainly focused on the hypothalamuspituitary-adrenal (HPA) axis as the key effector of the 
stress response. Selye also introduced the term "general adaptation syndrome" with its three phases : the alarm, resistance, and exhaustion stages (3). The intensity of the stress response might vary ; however, the stage of "alarm" characterized the neural and endocrine patterns would be essentially the same.

\section{Concept of "allostasis"}

Many current views concerning what stress means and how to define and approach it exist, but none has been widely accepted. Recently, McEwen (4) introduced the term "allostasis" into stress research. In contrast to homeostatic systems such as blood oxygen, blood $\mathrm{pH}$, and body temperature, which must be maintained within narrow ranges, allostatic regulations are broader and do not depend on set-point mechanisms, signals are not constant, and anticipation of need is an important element. Another aspect of this theory is that allostatic load also reflects aspects of lifestyle (e.g., eating a high-fat diet, lack of exercise, etc.) and disturbances of diurnal rhythms (e.g., sleep deprivation) that result from overexposure of various tissues to stress mediators. The most common allostatic responses in- volve the sympathetic nervous systems and the HPA axis. Allostatic systems enable us to respond to our physical states (e.g., awake, asleep, supine, standing, exercising) and to cope with noise, crowding, isolation, hunger, extremes of temperature, danger, and microbial infection. In response to a stressor, the body turns on an allostatic response that initiates a complex adaptive pathway, and then shut off this response when the threat is past, the infection is contained, the living environment is improved, or the speech has been given (Fig. 1A). However, if the inactivation is inefficient, there is overexposure to stress hormones. Longer-term exposure to increased secretion of stress hormones results in allostatic load and its pathophysiologic consequences. According to the allostasis theory, pathological stress responses can be classified into three types of allosteric load (Fig. 1). In the first type of abnormal adaptive response (Fig. 1B), adaptation to repeated stressors of the same type is lacking, resulting in prolonged exposure to stress hormones, as was the case for some of the people subjected to the repeatedpublic-speaking challenge (5). In the second type of abnormal load (Fig. 1C), there is an inability to shut
A. Normal

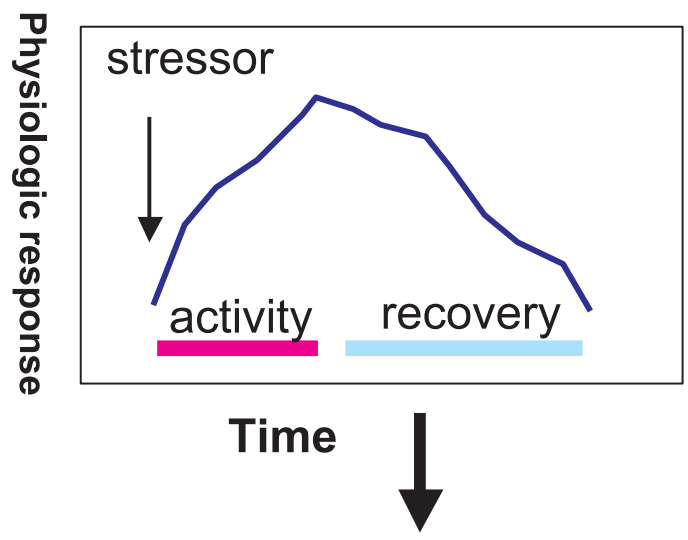

C. Prolonged response

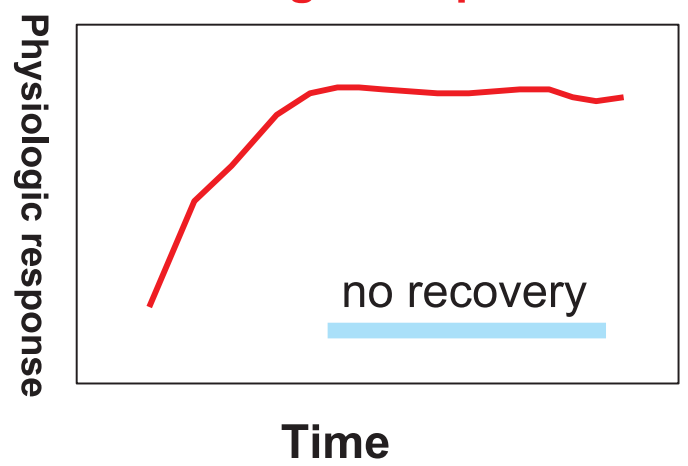

B. Lack of adaptation

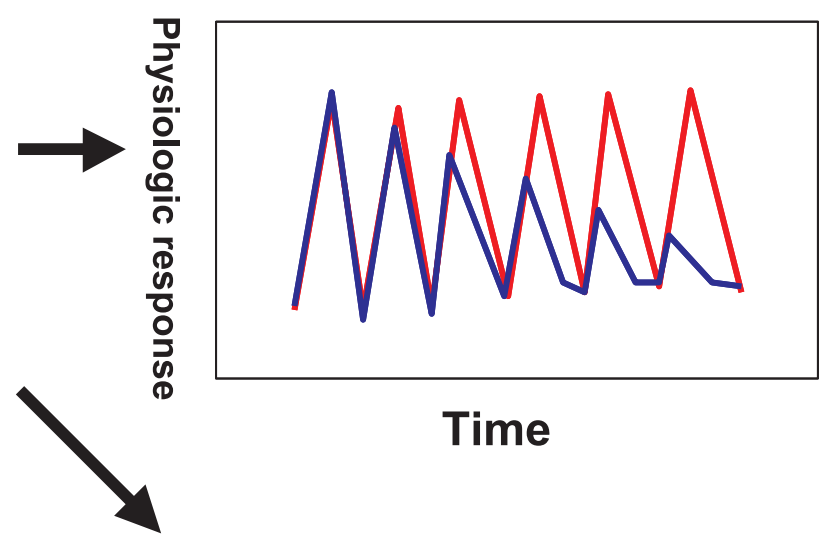

D. Inadequate response

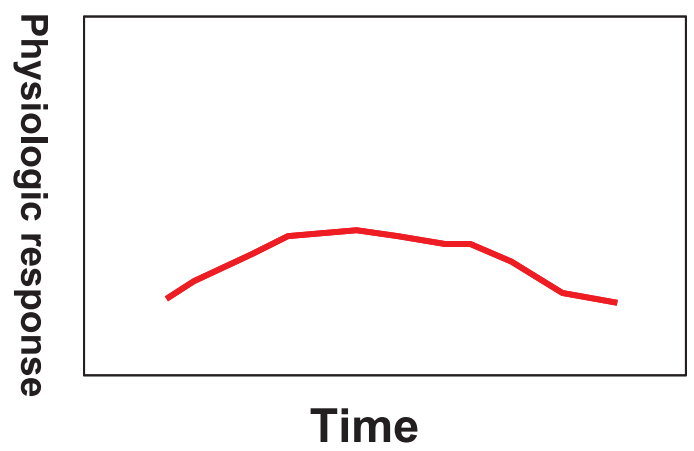

Fig.1. Three types of pathological stress response (allostatic load). Normal allostatic response is initiated by a stressor, sustained for an appropriate time, and then terminated (A). Abnormal stress responses are classified into lack of adaptation to the repeated exposures to the same stressor (B), prolonged response due to impairment of negative-feedback systems (C), and inadequate response that leads to compensatory hyperactivities of other mediators (D). These figures are modified from the data described in ref. 4 . 
off allostatic responses after a stress is terminated. Impaired negative-feedback pathways results in prolonged exposure to cortisol or cathecolamines, which may accelerate progressive structural damage and functional impairment. One speculation is that this type of allostatic load over a lifetime may cause the allostatic systems to wear out or become exhausted (6). A vulnerable link in the regulation of the HPA axis and cognition is the hippocampal region. The hippocampus play a crucial role in turning off the HPA axis. According to the "glucocorticoid-cascade hypothesis", wear and tear on this region of the brain leads to dysregulation of the HPA axis and cognitive impairment (7), which is associated hippocampal damage (8). In the third type of allostatic load (Fig. 1D), impaired responses by some allostatic systems trigger compensatory increases in others. When one system does not respond adequately to a stressor, the activity of other systems increases, since the underactive system is not providing the usual counterregulation. For example, if cortisol secretion does not increase in response to stress, secretion of inflammatory cytokines (which are counterregulated by cortisol) increases and results in an enhanced inflammatory response.

\section{THE HPA AXIS}

\section{Regulation of the HPA axis}

To understand the underlying mechanism for pathological allostatic loads, it is particularly important to reveal the complex networks for regulation of the HPA axis (Fig. 2). Corticotropin-releasing hormone (CRH) plays a central role in the regulation of the HPA axis, i.e., the final common pathway in the stress response. The action of $\mathrm{CRH}$ on adrenocorticotropic hormone (ACTH) release is strongly potentiated by vasopressin that is co-produced in increasing amounts when the hypothalamic paraventricular neurons are chronically activated. Whereas vasopressin stimulates ACTH release in humans, oxytocin inhibits it. ACTH release results in the release of corticosteroids from the adrenal that, subsequently, through mineralocorticoid and glucocorticoid receptors, exert negative feedback on the hippocampus, the pituitary, and the hypothalamus. The most important glucocorticoid in humans is cortisol, present in higher levels in women than in men. The inability to cope with stressful life events, which leads to the hypersecretion of corticosteroids, causes an increased risk for depression, obesity, osteoporosis, and cardiovascular disorders (9). The hippocampus expresses glucocorticoid re-

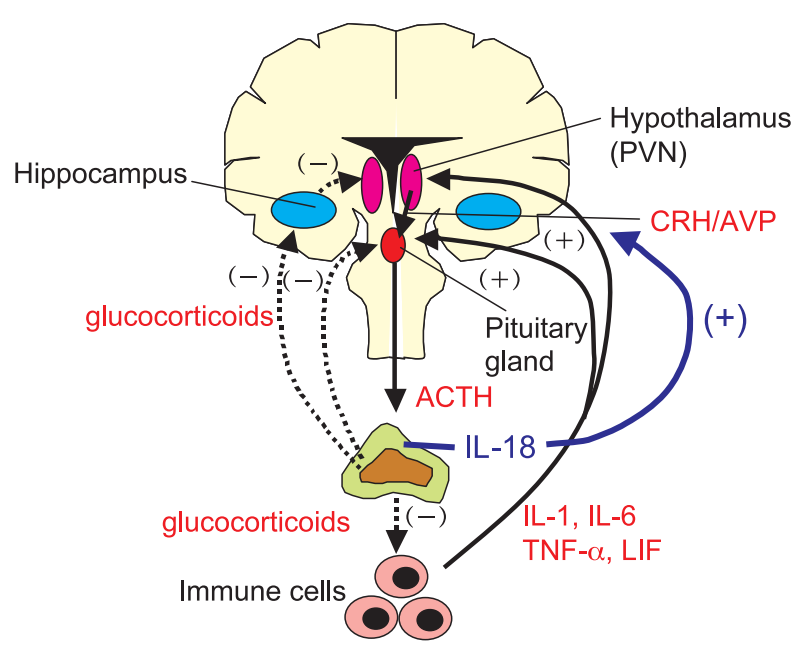

Fig.2. Organization, function, and regulation of the HPA axis. Glucocorticoid production and release from the adrenal glands is stimulated upstream regulators including corticotropin-releasing hormone (CRH) and arginine vasopressin (AVP) from the paraventricular nucleus (PVN) of the hypothalamus and adrenocorticotropic hormone $(\mathrm{ACTH})$ from the pituitary. Proinflammatory cytokines, such as interleukin-1 (IL-1), interleukin-6 (IL-6), tumor necrosis factor- $\alpha$ (TNF- $\alpha$ ), leukemia inhibitory factor (LIF) are also potent stimulators of $\mathrm{CRH}$ and thus hypothalamic-pituitary-adrenal (HPA) axis activity and glucocorticoid release. Glucocorticoids regulate their own production through negative-feedback pathways at the levels of the hippocampus, hypothalamus (CRH), and pituitary (ACTH) as well as the immune system (proinflammatory cytok ines). Glucocorticoid receptors in the hippocampus play a crucial role in turning off the HPA axis. Recently, ACTH-mediated production of IL-18 from the adrenal cortex has been shown to trigger IL- 6 production and possibly to participate in the positive-feedback pathway (20).

ceptor (GR), responds to increased glucocorticoids under stressful situations, and exerts an important, mostly inhibitory influence on the activities of the amygdala, the paraventricular nulei (PVN), the locus ceruleus, and noradrenergic cell groups. The impairment of this negative feedback system is implicated in the pathogenesis of stress-related disorders, particularly in major depression $(10,11)$. Hypercortisolaemia that is associated with coping problems is primarily related to emotional arousal, psychotic symptoms, and cognitive impairment (12). Hypercortisolaemia that occurs secondarily in patients with Cushing disease or after prolonged glucocorticoid administration causes similar symptoms. Research in nonhuman primates has suggested that stress and prolonged glucocorticoid exposure may damage the hippocampus $(13,14)$, thus raising the possibility that traumatic stress or the chronic stress of having post-traumatic stress syndrome (PTSD) may induce hippocampal degeneration in human. Studies examining postmortem tissue from suicide victims and individuals with major depression have revealed decreased GR mRNA expression in the hippocampus and cortex (15). Further- 
more, GR mRNA expression and its hormon-binding activity are both increased after treatment with antidepressants (16). Recently, it has been shown that acquired defect of forebrain GR produces depressionlike changes in adrenal axis regulation and behavior (17). Thus, dysregulation of the HPA axis may be a primary factor in the pathogenesis of stress-related disorders including major depression.

\section{Neuro-immune-endocrine interactions}

The central nervous system (CNS), the endocrine system, and the immune system are complex systems that interact with each other. Modulation of the immune response by the CNS is mediated by a complex network of bidirectional signals between the central nervous, endocrine, and immune systems (Fig. 2). For example, the catecholamines (adrenaline and nora drenaline), ACTH, cortisol, growth hormone and prolactin are all influenced by negative events and negative emotions, and each of these hormones can induce quantitative and qualitative changes in immune function. Depression can substantially boost cortisol levels, and increases in cortisol levels can provoke multiple adverse immunological changes (18). At the same time, numerous other cytokines including IL-1 are capable of influencing HPA secretory axis activity, with most having a stimulatory action (19). Cytokine receptors are localized to many neuroendocrine tissues. The HPA axis exposure to cytokines is not restricted to those carried within the vascular supply, since the CNS, pituitary, and adrenal synthesize a variety of cytokines and express their receptors at varied extents. Furthermore, cytokine regulation of the HPA axis occurs not only during infection, inflammation, and trauma, but also during periods of psychological and/or physical stress unrelated to the presence of tissue disease or damage. The pituitary and adrenal glands represent potential targets of cytokine action on the HPA axis when these organs are exposed to prolonged elevated cytokine levels. However, either direct or indirect stimulation of hypothalamic $\mathrm{CRF}$ secretion is the primary means by which cytokines (at least IL-1, IL-6, and TNF- $\alpha$ ) activate the HPA axis. Since IL-1 induces the secretion of IL-6, IL-6 may be, at least partly, responsible for activation of the HPA axis. However, elaboration of a single cytokine in response to a homeostatic threat is an unlikely event. Furthermore, there are no systematic studies of the mechanisms by which multiple cytokines (e.g., IL-1 plus IL-6) may induce HPA axis activation.

With regard to the interaction between cytokine and the HPA axis, Sekiyama et al. have recently shown a crucial role of IL-18 in this interaction (20). Mice exposed to immobilization stress causes elevation of both pro-IL-18 in the adrenal gland and mature IL-18 in plasma. ACTH is involved in the elevation of pro and mature IL-18, and superoxide-dependent activation of caspase 1 is likely to trigger the production of IL-18 (Fig. 3A). Interestingly, mice deficient of IL-18 fail to increase IL-6 when exposed to immobilization stress (Fig. 3B). These results have provided two important mechanisms of acute psychological stress. First, production of superoxide anion may trigger the acute stress response. Second, IL-18 may play a crucial role in the initiation of stress-triggered cytokine production (20). Further studies will reveal the IL-18-mediated regulation of the HPA axis, which may open a new field of research interest in the pathogenesis of stress-related disorders.

B.

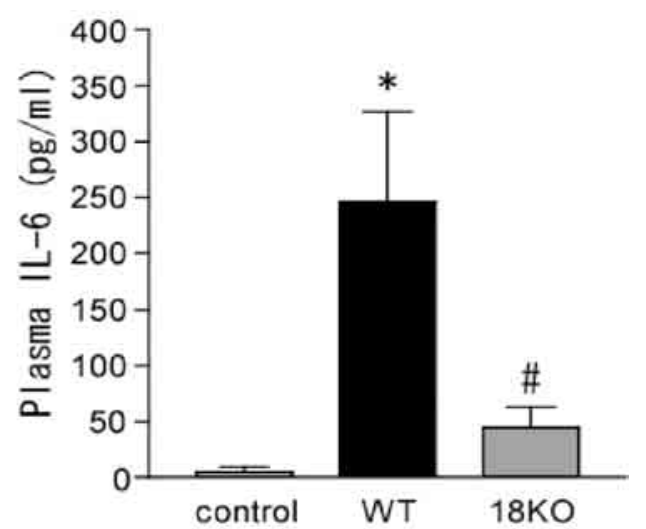

Fig.3. Effect of an immobilization stress on interleukin-18 (IL-18) levels in mouse plasma. Mice exposed to immobilization stress increase the plasma IL-18 level, which is blocked by administration of superoxide dismutase (SOD) (A). IL-6 production stimulated by immobilization stress is absent in IL-18 deficient mice (18KO). Values are means $\pm \mathrm{SD}, \mathrm{n}=9$. ${ }^{*} P<0.001$, compared with untreated control mice. $\# P<0.01$, compared with stressed wild mice. These figures are modified from the data described in ref. 20. 


\section{NEW STRATEGY FOR ASSESSMENT OF STRESS RESPONSE}

\section{Development of a stress DNA chip}

The microarray is now recognized as a useful clinical device to make diagnostic, therapeutic, or prognostic decisions for patients. The examples of special note are applications in the differential diagnosis of adult acute leukemias (21) and the identification of clinicaloutcome predictors in adult acute myeloid leukemia (22) and breast cancer $(23,24)$. In addition to these applications, high-throughput analysis of gene expression by microarray may have a potential advantage of being able to study complex responses, such as psychological stress response, in which the measurement of limited numbers of gene products does not always reflect the status. There were several classical approaches from social aspects or personal traits to assess stress. There are also physiological, biochemical, and clinical approaches for evaluation of stress response. But all of these methods take time and are not suitable to objectively assess the complex response regulated by the brain, immune, and endocrine systems. Unfortunately, at present there is no method to correctly and objectively assess the stress response. Now it is known that a number of stress-responsive genes including those with unknown functions are involved in the adaptive response. We are preferentially using a DNA microarray to objectively assess the complex response. Particularly, microarray is suitable for detection of abnormal stress response that may be related to the development of psychiatric disease or mental disorders. In addition to measurement of the levels of stressrelated hormones, their receptors, signal transduction molecules, and transcription factors, many studies on the polymorphism of functionally key genes that may participate in the development of mental disorders are currently underway. Proteomics analysis of stressresponsive protein markers is also available. Compared to these methods, the microarray technique is, however, well-established, powerful, and practical one.

We had developed a unique DNA microarray to specifically detect stress response in peripheral leukocytes, collaborating with Hitachi Life Science Group (Table 1). We selected 1,467 genes that are categorized into stress hormones, neurotransmitters, cytokines, chemokines, growth factors, and receptors for these stress mediators, signal transduction molecules, transcription factors, heat shock proteins, cell cycle- or growth-related molecules, apoptosis-related molecules, and others. We confirmed that transcripts for these genes were actually amplified by PCR using peripheral
Table 1. Characteristics of a stress DNA chip

1. Stress DNA chip carries cDNAs or 80 mer oligonucleotides for 1,467 genes, including stress hormones, neurotransmitters, cytokines, chemokines, growth factors, receptors for these ligands, signal transduction molecules, transcription factors, heat shock proteins, cell cycle- or growth-related molecules, apotosis-related molecules, and others.

2. Stress DNA chip needs only $2.5 \mathrm{ml}$ of peripheral blood for analysis.

3. Stress DNA chip is not a tool for genetic diagnosis.

4. Stress DNA chip is highly reproducible (CV values, $<20 \%$ for cDNA chip and $<10 \%$ for oligoDNA chip; dynamic range, three orders of magnitude).

5. Stress DNA chip is a powerful tool for assessment of mental status.

leukocytes from healthy volunteers. This microarray (named as stress DNA chip) needs only $2.5 \mathrm{ml}$ of peripheral blood for analysis. It is not a genetic diagnosis. This method is highly reproducible and a powerful tool for evaluation of complex stress response. Actually it is impossible to examine the gene expression profile in the brain of living subjects. Peripheral leukocytes express most receptors for stress-related or stressregulating molecules. We decided to detect gene expression profile that may indirectly assess the event in the CNS. With cluster analysis, data mining, and bioinformatics, we can select around three hundred genes that can be used for assessment of mental status.

Gene expression profile in peripheral leukocytes from healthy students exposed to life event stress

We applied this microarray for assessment of stress response in students of our medical school, who received an examination to enter into our graduate school (25). We collected the control blood one day before the examination. After a 6 -hour paper test, changes in the mRNA expression of 1,467 genes were examined (Fig. 4). Only one student (student 2) responded to the lifeevent stress, while the others did not. These results suggest that gene expression profiling is a reliable method to find out individuals highly responsive to stressful life events.

This method was also applied to examine stress response of graduate students subjected to one of the most stressful events for them. We collected control blood samples one month before the official examination for their $\mathrm{PhD}$, and then we examined the change in gene expression profile in peripheral leukocytes 2 hours before, or 2 and 24 hours after the oral presentation. As shown in Fig. 5, the individual responses varied. One student (student 9 ) was highly responsive and showed a marked change, whereas for other students (students 1,4 , and 6 ), the event was not so se- 

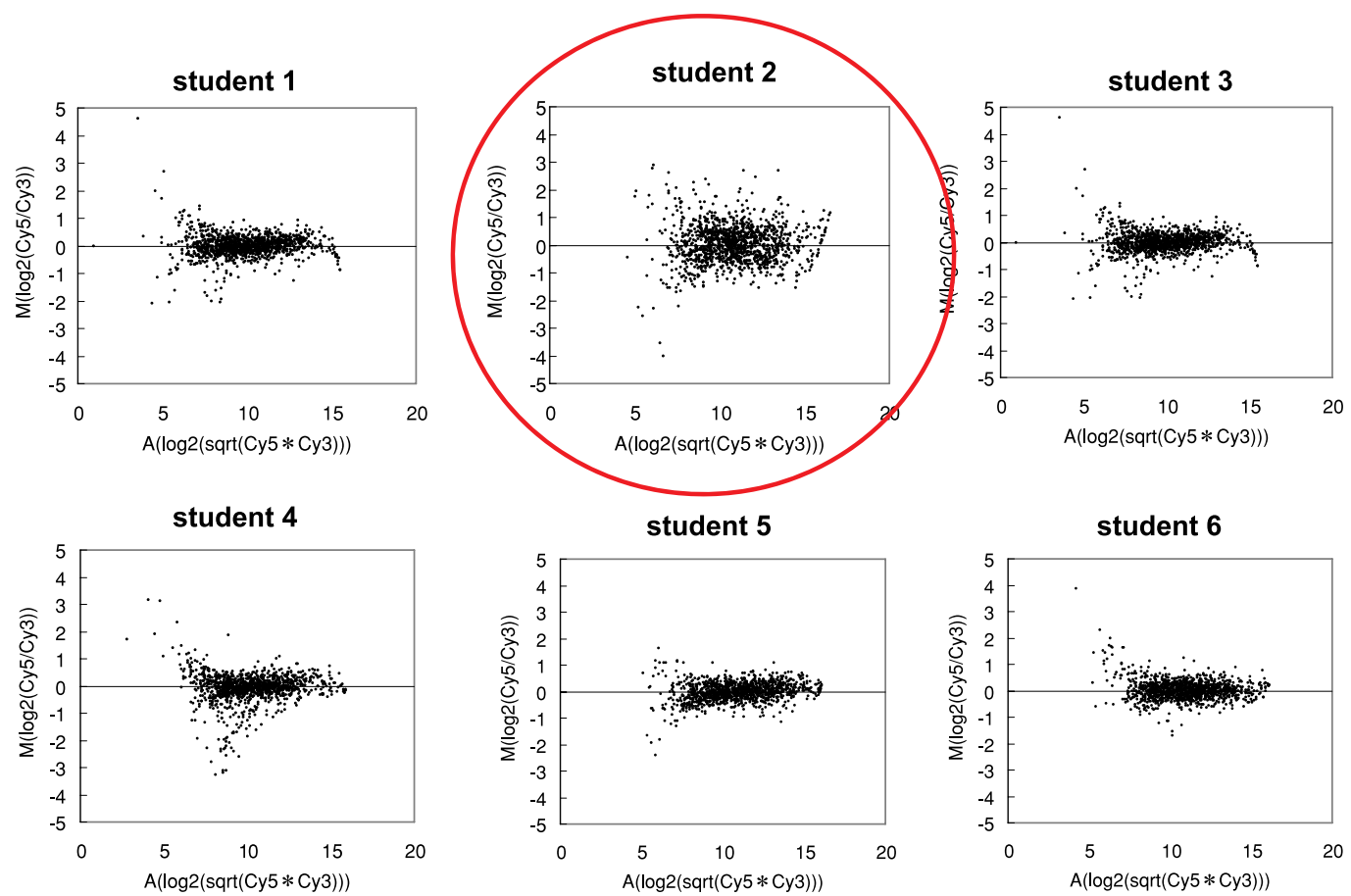

Fig.4. Changes in gene expression in peripheral leukocytes from healthy students taking entrance examination for graduate school. Control blood was collected from 6 healthy university students one day before the entrance examination for graduate school. After a 6 -hour paper test, changes in the mRNA expression of 1,467 genes were examined. Individual response is shown by scatter plots.

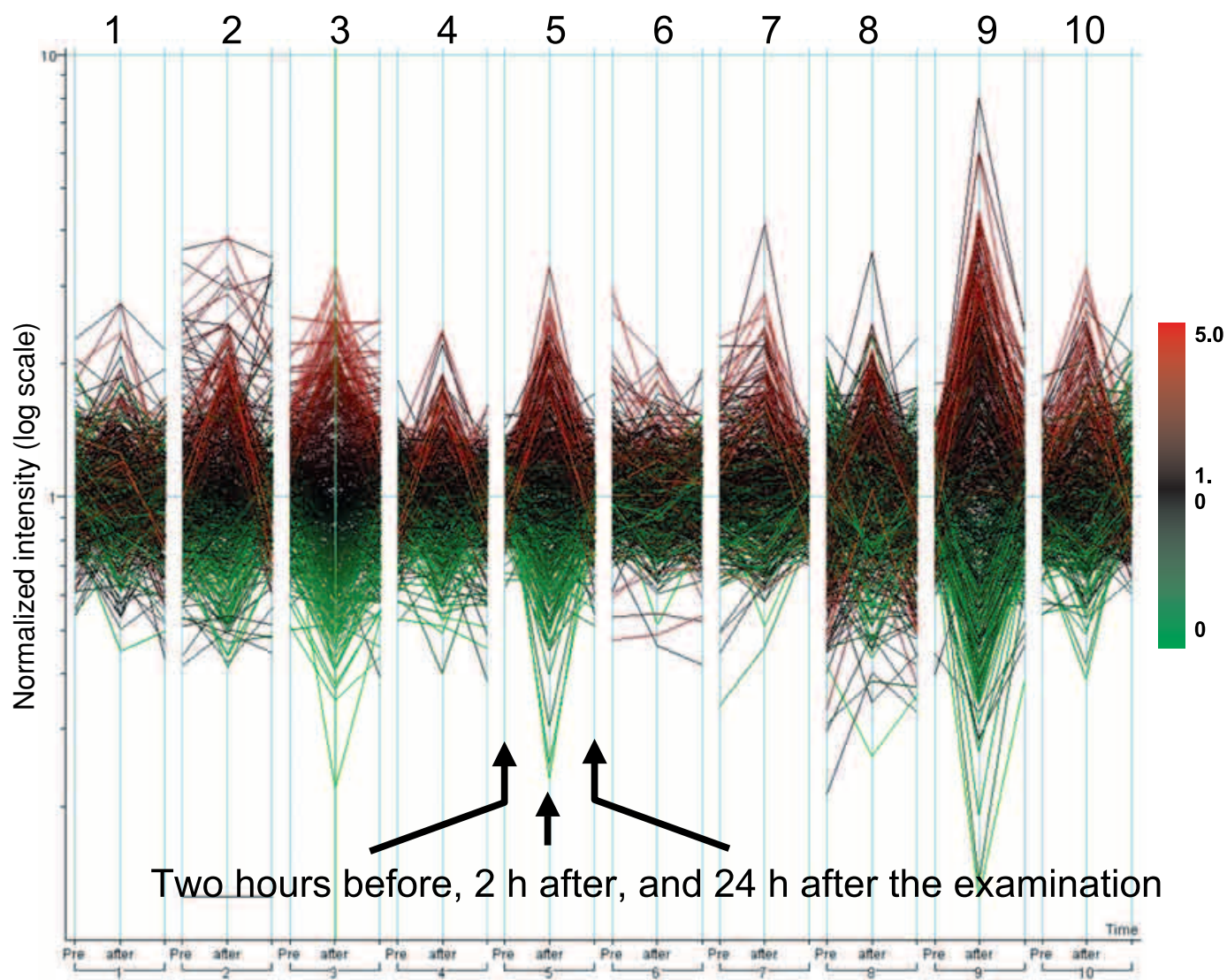

Fig.5. Time courses of gene expression values in peripheral leukocytes from 10 healthy students during stress exposure. After 10 students of postgraduate school passed the initial screening of their $\mathrm{PhD}$ manuscripts, they took the final examination, which consisted of an oral presentation of the $\mathrm{PhD}$ theses and a question-and-answer session. Venous blood (10 ml) was taken from each subject $2 \mathrm{~h}$ before and 2 or $24 \mathrm{~h}$ after the examination. The sample collected 4 wk before the presentation was used as a reference. All blood samples were collected under fasting conditions. Time-dependent changes in 519 mRNA levels $2 \mathrm{~h}$ before and $2 \mathrm{~h}$ or $24 \mathrm{~h}$ after the examination are shown. 
rious. The other student (student 2), for example, was already stressed before the presentations, and stressrelated changes in gene expression still continued 24 hours after the presentation. Using this technique, we can clearly detected individual variations with regard to the magnitude and time course of the stress response.

A cluster analysis of the gene expression patterns in 10 students provided very important findings (Fig. 6). In spite of individual variations in magnitude and time course, a distinct group of genes are involved in this response. A group of genes are up-regulated, while another group of genes are down-regulated. Situations and performances might be different among the sub-

\section{0}

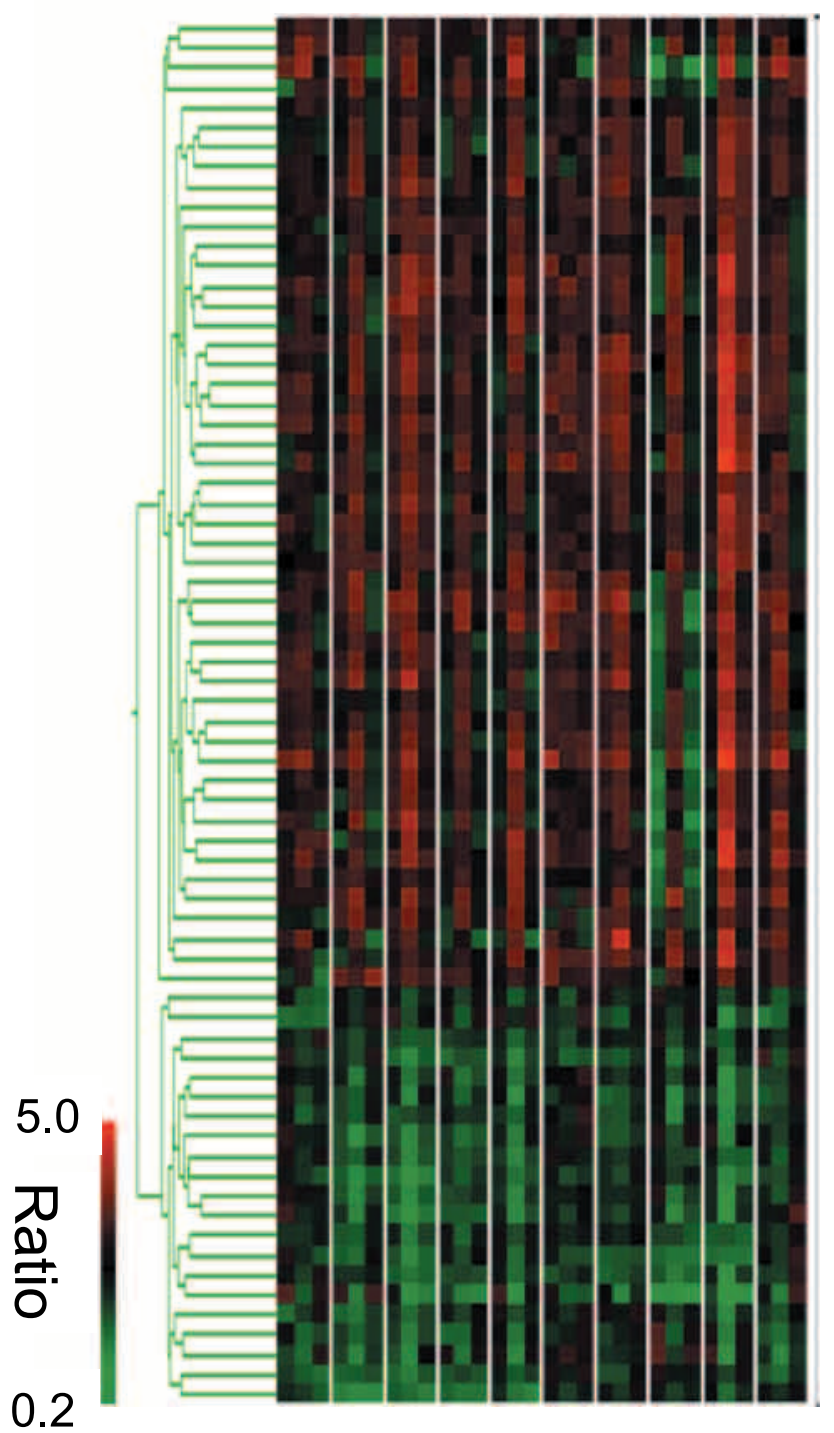

Fig.6. Hierarchical clustering of significantly responsive genes to psychological stress. Changes in gene expression in leukocytes from 10 students of graduate school were examined as described in the legend to Fig. 5. Significantly responsive genes were analyzed by the Bayesian $t$-test at $P<0.05$. The results of hierarchical clustering for the responsive 70 genes among 10 healthy subjects are displayed with a gene tree Individual genes are listed in ref. 25. jects: some students exhibited good performances, while others did not. In spite of the individually different backgrounds, only a distinct group of genes uniformly up-regulated or down-regulated are involved in the response to the life event stress. We could select 70 genes that were significantly responsive genes (Fig. 6). We also confirmed that expression of these 70 genes was not significantly changed during the daily activities, suggesting that the selected 70 genes are useful maker genes for assessment of acute psychological stress in healthy subjects.

\section{Future applications of stress DNA chip}

We are also using this technique for diagnosis of major depression and found that patients with depression had unique gene expression profiles in peripheral leukocytes. From our preliminary studies, we have identified a group of trait-related genes, a group of staterelated genes, and maker genes for sub-grouping patients with major depression. These maker genes may be useful for diagnosis, or assessments of disease status, therapy, and possibly prognosis of major depression. We also found the important evidence that only one gene overlaps between stress-responsive genes in healthy subjects and depression-related genes. Furthermore, this gene changes its expression into opposite direction between the two groups. These results suggest that the patient with depression shows abnormal stress response. Thus, in addition to the measurement of magnitude and duration of stress response, the qualitative analysis of the response is likely to be essential for detecting disease-associated stress response. For this purpose, a simple microarray carrying only several hundred genes is enough for detection of the pathological stress response. Now we are making a new microarray carrying a limited number of maker genes.

\section{ACKNOWLEDGEMENT}

This study was supported by a Grant of the Special Coordination Funds for Promoting Science and Technology from the Ministry of Education, Culture, Sports, Science and Technology of Japan (to K. R.), a Grantin-Aid for the 21st Century COE program, Human Nutritional Science on Stress Control, from the Ministry of Education, Culture, Sports, Science and Technology of Japan, and a Grant from the Research Institute of Science and Technology for Science of Japan Science and Technology (to K. R.). 


\section{REFEREMCES}

1. Cannon WB : Organization for physiological homeostasis. Physiol Rev $9: 399-431,1929$.

2. Selye $\mathrm{H}: \mathrm{A}$ syndrome produced by diverse nocious agents. Nature $138: 32,1936$.

3. Selye $\mathrm{H}$ : The physiology and pathology of exposure to stress. A treatise based on the concepts of the general adaptation syndrome and the diseases of adaptation. Acta Inc, Montreal, 1950.

4. McEwen BS : Protective and damaging effects of stress mediators. N Engl J Med 338: 171-179, 1998.

5. Kirschbaum C, Prussner JC, Stone AA, Federenko I, Gaab J, Lintz D, Schommer N, Hellhammer DH : Persistent high cortisol responses to repeated psychological stress in a subpopulation of healthy men. Psychosom Med 57 : 468-474, 1995.

6. Seeman TE : Robbins RJ. Aging and hypothalamicpituitary-adrenal response to challenge in humans. Endocr Rev 15 : 233-260, 1994.

7. Sapolsky RM, Krey LC, McEwen BS: The neuroendocrinology of stress and aging : the glucocorticoid cascade hypothesis. Endocr Rev 7 :284-301, 1986.

8. Meaney MJ, Aitken DH, van Berkel C, Bhatnagar S, Sapolsky RM : Effect of neonatal handling of agerelated impairments associated with the hippocampus. Science 239 : 766-768, 1988.

9. Brown ES, Varghese FP, McEwen BS: Association of depression with medical illness : does cortisol play a role? Biol Psychiatry 55 : 1-9, 2004.

10. Raison CL, Miller AH : When not enough is too much : the role of insufficient glucocorticoid signaling in the pathogenesis of stress-related disorders. Am J Psychiatry 160 : 1554-1565, 2003.

11. de Kloet ER, Joëls M, Holsboer F : Stress and the brain: from afaptation to disease. Nat Rev Neurosci 6 : 463-475, 2005.

12. Lupien SJ, de Leon M, de Santi S, Convit A, Tarshish C, Nair NP, Thakur M, McEwen BS, Hauger RL, Meaney MJ : Cortisol levels during human aging predict hippocampal atrophy and memory deficits. Nature Neurosci 1:69-73, 1998.

13. Sapolsky RM, Uno H, Rebert CS, Finch CE : Hippocampal damage associated with prolonged glucocorticoid exposure in primates. J Neurosci $10:$ 2897-2902, 1990.

14. Gould E, Tanapat P, McEwen BS, Flugge G, Fuchs E : Proliferation of granule cell precursors in the dentate gyrus of adult monkeys is diminished by stress. Proc Natl Acad Sci USA 95 : 31683171, 1998.

15. Modell S, Yassouridis A, Huber J, Holsboer F :
Corticosteroid receptor function is decreased in depressed patients. Neuroendocrinology 65 : 216-222, 1997.

16. Pariante CM, Miller AH : Glucocorticoid receptors in major depression : relevance to pathophysiology and treatment. Biol Psychiatry $49: 391-404,2001$.

17. Boyle MP, Brewer JA, Funatsu M, Wozniak DF, Tsien JZ, Izumi Y, Muglia LJ : Acquired deficit of forebrain glucocorticoid receptor produces depression-like changes in adrenal axis regulation and behavior. Proc Natl Acad Sci USA 102 : 473478, 2005.

18. Glaser R, Kiecolt-Glaser JK : Stress-induced immune dysfunction : implication for health. Nat Rev Immunol 5 : 243-251, 2005.

19. Turnbull AV, River CL: Regulation of the Hypothalamicpituitary-adrenal axis by cytokines : Actions and mechanisms of action. Physiol Rev 79 : 1-71, 1999.

20. Sekiyama A, Ueda H, Kashiwamura S, Sekiyama R, Takeda M, Rokutan K, Okamura H : A stressinduced, superoxide-mediated caspase-1 activation pathway causes plasma IL-18 upregulation. Immunity 22 : 669-677, 2005.

21. Bullinger L, Döhner K, Bair E, Fröhling S, Schlenk RF, Tibshirani R, Döhner H, Pollack JR: Use of gene-expression profiling to identify prognostic subclasses in adult acute myeloid leukemia, N Engl J Med 350 : 1605-1616, 2004

22. Valk PJM, Verhaak RGW, Beijen MA, Erpelinkck CAJ, Barjesteh van Waalwijk van Doorn-Khosrovani S, Boer JM, Beverloo HB, Moorhouse MJ, van der Spek PJ, Löwenberg B, Delwel R: Prognostically useful gene-expression profiles in acute myeloid leukemia, N Engl J Med 350 : 1617-1628, 2004.

23. Hedenfalk I, Duggan D, Chen Y, Radmacher M, Bittner M, Simon R, Meltzer P, Gusterson B, Esteller M, Kallioniemi OP, Wilfond B. Borg A, Trent J : Gene-expression profiles in hereditary breast cancer, N Engl J Med 344 : 539-548, 2001.

24. Van De Vijver MJ, He YD, Van't Veer LJ, Dai H, Hart AAM, Voskuil DW, Schreiber GJ, Peterse JL, Roberts C, Marton MJ, Parrish M, Atsma D, Witteveen A, Glas A, Delahaye Van Der Verde LT, Bartelink H, Rodenhuis S, Rutgers ET, Friend SH, Bernards R : A gene-expression signature as a predictor of survival in breast cancer. N Engl J Med 347 : 1999-2009, 2002.

25. Morita K, Saito T, Ohta M, Ohmori T, Kawai K, Teshima-Kondo S, Rokutan K: Expression analysis of psychological stress-associated genes in peripheral blood leukocytes. Neurosci Lett 381 : 57-62, 2005. 